\title{
STATEMENT FROM THE EDITOR
}

This is the first issue of a new journal, (AIEDAM), that is intended to provide a forum that is both interesting and credible to two communities. One community consists of engineers, designers and scientists who see in the artificial intelligence (AI) technologies leverage that can be brought to bear on difficult technical problems. The other community is that collection of researchers in artificial intelligence and computer science who are interested in applications and who see in those applications the generation of stimulating AI research questions. We are thus interested in ecouraging the publication of articles that develop new and interesting applications which are firmly grounded in state-of-the-art AI research.

We shall be particularly receptive to articles from the fields of chemical, civil, industrial and mechanical (i.e. non-VLSI) engineering. To be more specific, areas of special concern center around the use of AI techniques in support of planning, design, finite element analysis, simulation, spatial reasoning, graphics, optimization and manufacturing. Particular theoretical areas of interest include the following:

* knowledge-based (expert) systems, including knowledge acquisition, knowledge representation, control strategies and system architectures;

* geometric and spatial reasoning, knowledgebased support of graphics and solid modeling;

* reasoning under uncertainty, integration of decision support technologies; and
* AI languages and machines, including exploratory programming environments and expert systems shells and tools.

We will publish original research articles of archival interest. We will also solicit comprehensive and thoughtful survey articles and tutorials in areas of particular interest to our readers. We also intend to develop the following sections:

Book Reviews (under the stewardship of Professor David Brown);

Research Briefs (with Professor Manjula Waldron as cognizant editor) to report (substantial) work in progress;

Announcements of meetings and symposia; and

Tools to provide information on new software, environments and hardware.

This journal is obviously a new venture, one that the members of the Editorial Board and the Publisher regard as exciting and challenging. We believe that we have assembled a distinguished Board; as Editor, I am grateful to our Board members and to Academic Press for contributing their insights, efforts and reputations to this endeavor. I invite you, the reader, to contribute not only research papers, but your comments and ideas on our directions and emphases.

Cuve L. Dym Editor

\section{Note regarding book reviews}

This journal is particularly concerned that we inform our readers of books that cover areas of interest to the journal. To this end, it is intended to provide book reviews on a regular basis and also to incorporate a list of books received for review.
For this to be as comprehensive as possible, the Book Reviews Editor wishes to be notified by publishers and authors of forthcoming titles and to receive from publishers copies of books for review. All relevant books will be included in the "books received 